\title{
A Survey on Automatic Question Paper Generation System
}

\author{
Amruta Umardand ${ }^{1}$, Ashwini Gaikwad ${ }^{2}$ \\ Student, Computer Science \& Engg, DACOE, Karad, India ${ }^{1,2,3,4}$
}

\begin{abstract}
In today's world education is the most important thing, and in that important part is examination process. So the generation of question paper is a difficult process for teachers and also this process was tedious and insecure. So we have presented forward technique that is an "Automatic Question Paper Generation System". Which is randomized, fast and also riskless. In that system, every task is performed by automated that include subject selection, question entry, paper management. This system randomly selects question like in categorized manner from the file and generate question paper. This system is useful for many educational institutes.
\end{abstract}

Keywords: Automation, paperless, question generation, randomization.

\section{INTRODUCTION}

In any educational field main goal is improve the quality • of student. Beforehand question papers are generated manually, teachers require more time and power to create examination paper because creating examination paper done by writer's knowledge, experience, and style and therefore we introduce new advanced technology that is the automatic question paper generation system. This requires less manpower, using this system to process different uncommon sets of papers automatically. The system takes over the entire difficult task and paperwork swiftly and efficiently. Question bank stored in a database based on subject, class, and section and weight age. The automatic paper generator is one and only system for creating question paper. This system performs all work related to paper creation. For example, work starting from choose question bank to create a paper. This system useful for both small and large institute.

The authorities have the flexibility to generate class test, unit test, terminal test and final test. We also allow admin to provide weight age and complexity for each of these questions. After these questions are stored in the database along with their weight age during the time of question paper generation the admin just has to select the percentage of difficulty. On this selection, the system selects questions randomly according to the difficulty that admin chooses. The questions are chosen based on their complexity level.

\section{- Randomization:}

Randomization is the select things in a random manner, which does not follow any deterministic pattern.

\section{- Automation:}

Automation means, which is not required any human intervention. In this technique, all processor work is done automatically. Using automation replace manual work with the electronic device due to this productivity will be increased and reduced working time.
Paper-based vs paperless:

If Create question papers using paper-based system then many problems we faced like:

- Low security

- Time-consuming.

- Repetition of a question may occur.

- Slow process.

If Create question paper using paperless system then overcomes above problems:

- Provide higher security.

- Faster because of fully depend on the computer.

- Randomized process.

- Automated process.

\section{Features:}

We did not need to collect the papers as built in ready to use question bank is available. Duplication of questions is avoided. There are three levels of difficulty is provided is difficult, medium and easy. The question paper is generated in less time and more accuracy. We can conduct the record of developed question paper. In this system, security is provided through the password. Only admin can develop and see the question paper.

\section{Advantages:}

- Large portions are covered by the system and generate paper skilfully.

- Higher security and encryption.

- Faster because it is computer based.

- Using this system we ensure that no chance of paper leaks.

- This system is totally random and unbiased.

- Using this system no requirement of transporting paper with the help of police security vans.

- With this system less human efforts time and resources. 
IARJSET

\section{Limitations:}

- Lack of storage space.

- Inefficient document transportation.

- One of the most important drawbacks of the system is if we not given paper inputs then produce wrong results.

- In this system occurs editing problems.

- Development cost is more

\section{LITERATURE SURVEY}

Manual generation of question paper by an individual is complex, a simple and efficient way for generating question papers is automatic question paper generation system [3]. In that select the questions according to patterns and prevent the repetition of questions. Then generate question papers in sorted order and stored as a file [3].The system performs many tasks in fasted way without any complexity. The question paper generation system uses question banks and different mathematical models [1].

CQG system (Cloze Question Generation)[1]. Containing sentences with one or more blanks and multiple choice questions. This system creates important close questions. This system has three modules: sentence selection, keyword selection, and distracter selection. Informative and relevant sentences are selected in the first stage. In second stage keywords are identified from selected sentence. In the final stage answer, alternative for the keywords is selected. The first stage is not domain specific and the last stage is domain specific. Evaluation of the CQG system is done by following three phases.

- Evaluation of selected sentence.

- Evaluation of selected keyword.

- Evaluation of selected distracters.

Automatic question generation on the basis of discourse connectives [2].using this system we automatically generate questions from natural language text using discourse connectives. This system is divided into two modules: Syllabus and Formation of semantic-based automatic question generation.

A Library Automation System utilized in University of Toronto Library in 1963-1972[4] and the University of Illinois at Urbana-Chamnpaign1965-2000[5] Automated System for Educational Assessment [3] developed in Nigeria, e-Learning System [6], Automated Project Grading and Instant Feedback System [7] are well-known examples of Automated Systems. In these examples, automation is achieved in their manual process and improves performance. In automation converting the paper based system into the paperless system [8].

Automatic question paper generator system [9] used a randomized technique. This system has modules like user administration, subject selection, difficulty level specification, question entry, question management, paper generation, and paper management. The system uses attributes such as storing question in database, admin selected complexity level of questions, maintenance of the database.

"Framework for Automatic Examination Paper Generation System ${ }^{\text {ee }}$ this paper provided the process to generate automatic question paper [1]. Manually generation of question paper is quite a difficult task. This system provided a simple and efficient way for an examination paper generations. A three-tier model is provided in this framework [1].

Generation of Examination Papers is managed by the Syllabus Engine, Pattern Composer, and Question Aggregator. The system is based on the pattern or skeleton of the course. In the system, questions are entered through the Question Aggregator. Weight age, the difficulty level of question and marks these are the attributes of question paper.

\section{PROPOSED SYSTEM}

Steps:

- Start the application.

- Admin will add the professor to the database and give login id and password.

- System check login is done by authorized user.

- After successful verification, it asks for a number of questions to be inserted in the question paper.

- Questions inserted by the professor are stored into the created database.

- By entering the department, semester, subject, weight age professors can generate question paper.

- When professor clicks on the "Generate Paper" button then the paper will be generated.

B. Modules in Automatic question Paper Generator

- Login Module

- Administrator Module

- Teacher Module

\section{Login Module:}

This module subdivided into Teacher and Administrator. In this admin as well as teacher enters their id and password to access the system.

\section{Administrator Module:}

This module has two subparts User Management and Question Management. In first module admin will logged and add professor to that system and admin send id and password to the added or selected teacher to access this system and all details of teacher stored in database. In Next module that is Question Management set question paper as per department, subject, examination type, complexity level.

Teacher module divided into: Question Entry, Test Paper Customization, Generate System and Previously Generated Test Papers. In first module teacher create 
IARJSET

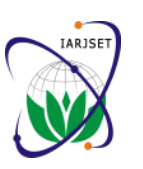

International Advanced Research Journal in Science, Engineering and Technology

National Conference on Innovative Applications and Research in Computer Science and Engineering (NCIARCSE-2017)

AGTI's Dr. Daulatrao Aher College Engineering, Vidyanagar Extension, Karad

Vol. 4, Special Issue 4, January 2017

question paper as per department, Examination type, subject, question and marks and this all data stored in the database. Next module is Test Paper Customization system generate paper in txt format; at the time of examination teacher simply make changes in the test paper. After that, teacher generates paper by entering all required information. Next is Previously Generated Test paper teacher login to the system to examine previous test paper in this way to avoid the duplication.

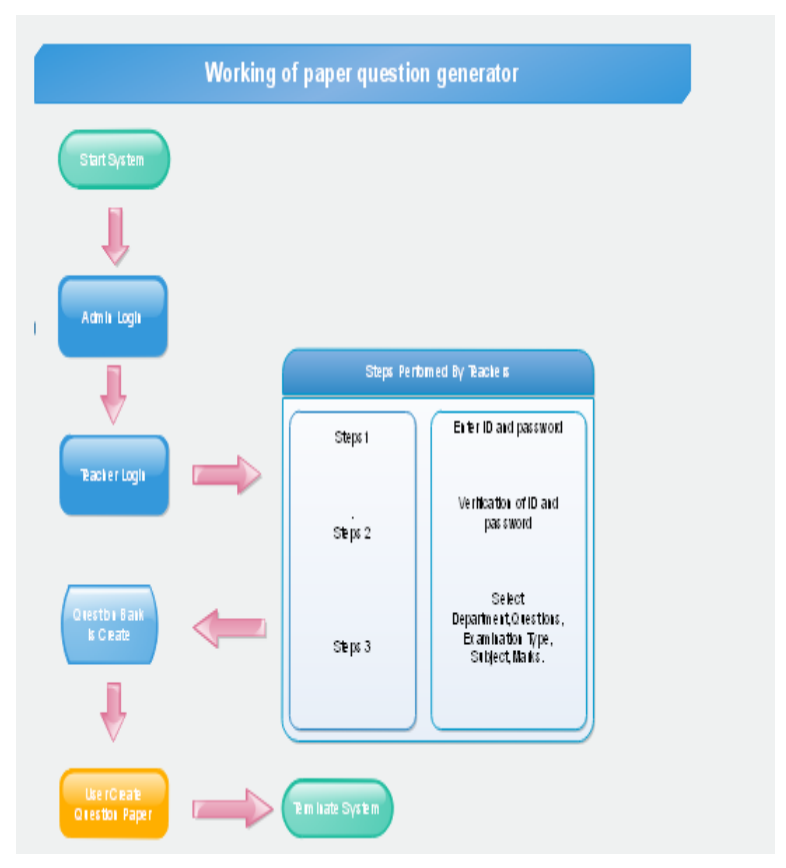

IV.CONCLUSION

This system represents for generating automation in examination process as the real-time application, with the help of system easily generates automatic question papers and improvement from the traditional method of paper generation. This system is web-based system and also computer based. In this system, we have implemented randomized process so avoid the repetition of questions. This system platform is very secure and also faster because it is computer and web-based system.

\section{ACKNOWLEDGMENT}

It gives us great pleasure in presenting a paper titled "Automated Question Paper Generation System". We wish to thanks all the people who gave valuable guidance and support to complete this paper. We especially thank our Prof. Sayali Shinde who gives us guidance about the creation of survey paper generation and most important they gave encouragement up to completion of the paper. We also thankful to our head of the department Computer Science and Engineering Prof. Ashish Patil sir to encourage us. And We would like to thanks to our whole CSE department. We are extremely thankful to our all teachers of our college.

\section{REFERENCES}

[1] Ashok Immanuel and Tulasi. B, "Framework for Automatic Examination Paper Generation System," International Journal of Computer Science Trends and Technology, vol. 6, issue 1, Jan March 2015.

[2] Automatic Cloze-Questions Generation by Annamaneni Narendra, Manish Agarwal and Rakshit shah LTRC, IIIT-Hyderabad, India.

[3] Automatic Question Generation using Discourse Cues by Manish Agarwal_, Rakshit Shah_and Prashanth Mannem.

[4] Ritvars Bregzis, Calvin Gotlieb,Carole Moore, "The Beginning of Automation in the University of Toronto Library, 1963-1972",in IEEE Annals of the History of Computing, April-June 2002.

[5] Prof. Godswill Obioma Prof. Ismail Junaidu , Dr. Grace Ajagun, "The Automation of Educational Assessment in Nigeria: Challenged and Implications for Pre-Service Teacher Educaion", Annual Conference of the International Association for Educational Assessment (IAEA) held at the Dan Panorama Hotel, Tel-Aviv, IsrealOctober 20th -25th, 2013

[6] M. Jou,J.K. Shiauand H.W. Zhang,"Application of Web Technologies in Automationon Technology Education", International Journal of Computers and Applications, Vol.31, No. 4, 2009

[7] Xiang Fu, Boris Peltsverger, Kai Qian, Lixin Tao, Jigang Liu," APOGEE -Automated Project Grading and Instant Feedback System for Web Based Computing", Computer Science and Information Technology, 2nd IEEE International Conference 2009.

[8] Gerald Weber," Defining the Paperless Workplace with the Paper Metaphor -Not a Contradiction in Terms", Conference: Proceedings of the Fourth Australasian Workshop on Health Informatics and Knowledge Management -Volume 120.

[9] Kapil Naik, Shreyas Sule, Shruti Jadhav and Surya Pandey, "Automatic Question paper Generation System using randomization algorithm" IJETR, Vol.2, Issue 12, pp.1-3, Dec 2014.

[10] Surbhi Choudhary, Abdul Rais Abdul Waheed. Shrutika Gawandi and Kavita Joshi, "Question Paper Generator System," International Journal of Computer Science Trends and Technology, vol. 3, issue 5, Sept-Oct 2015

\section{BIOGRAPHIES}

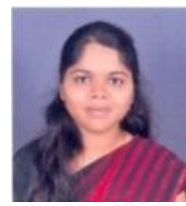

Ms. Amruta Nilkanth Umardand Department of Computer Science and Engineering from AGTI's Dr. Daultrao Aher College of Engineering, Karad

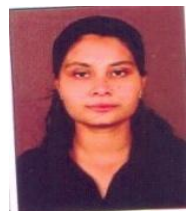

Ms. Ashwini Gyaneshwar Gaikwad, Department of Computer Science and Engineering from AGTI's Dr.Daultrao Aher College of Engineering, Karad. 